\title{
Quantum dot energy relaxation mediated by plasmon emission in doped covalent semiconductor heterostructures
}

\author{
A. V. Fedorov,* A. V. Baranov, and I. D. Rukhlenko \\ Saint-Petersburg State University of Information Technologies, Mechanics and Optics, \\ 49 Kronverksky Avenue, 197101 St. Petersburg, Russia \\ T. S. Perova \\ Department of Electronic and Electrical Engineering, Trinity College, University of Dublin, Dublin 2, Ireland \\ K. Berwick \\ Department of Electronic and Communications Engineering, Dublin Institute of Technology, Dublin 8, Ireland
}

(Received 14 February 2007; published 24 July 2007)

\begin{abstract}
The interaction between interface plasmons within a doped substrate and quantum dot electrons or holes has been theoretically studied in double heterostructures based on covalent semiconductors. The interface plasmon modes, the corresponding dispersion relationship, and the intraband carrier relaxation rate in quantum dots are reported. We find the critical points in the interface plasmon density of states for multilayered structures results in enhanced quantum dot intraband carrier relaxation when compared to that for a single heterostructure. A detailed discussion is made of the relaxation rate and the spectral position dependencies on the quantum dot layer thickness as well as on the dopant concentration. The material system considered was a $p-\mathrm{Si} / \mathrm{SiO}{ }_{2} /$ air heterostructure with Ge quantum dots embedded in an $\mathrm{SiO}_{2}$ layer. This structure is typical of those used in technical applications.
\end{abstract}

DOI: $10.1103 /$ PhysRevB.76.045332

PACS number(s): 78.66.Fd, 63.22.+m, 71.35.Cc, 78.55.Cr

\section{INTRODUCTION}

Continuing strong interest in semiconductor quantum dots (QDs) is not just due to their unique physical properties, but also due to the growing number of devices which utilize these properties. QDs are a key element in a wide variety of electronic and optoelectronic devices, including singleelectron transistors, ${ }^{1}$ quantum bits, ${ }^{2}$ memory cells, ${ }^{3}$ and lasers. ${ }^{4}$ The properties of these devices rely on the QD electronic subsystem relaxation efficiency; therefore, the study of carrier relaxation mechanisms in QDs is of central importance. A knowledge of these relaxation phenomena is particularly important since it is intended that many nanoelectronic devices will be incorporated into integrated circuits (IC). The characteristic distances between the structural elements in these ICs is expected to be several tens of nanometers. Strong interaction of QDs with other circuit elements, viz. doped substrates, buffer and wetting layers, semiconductor quantum wells and wires, is expected over these distances. This interaction would be expected to strongly influence the performance of the circuit, at both device and system level. A careful analysis of energy relaxation processes in QDs, induced by interactions occurring over characteristic distances of several tens of nanometers, is required.

To date, the influence of a variety of elementary excitations, either localized inside a QD or at its interface, on the relaxation processes of QD-based devices has been considered in a number of studies. The effects of confined acoustical 5 and optical phonons (including interface ones), ${ }^{5-15}$ plasmons, ${ }^{16-18}$ polaronlike states in QDs, ${ }^{7,19,20}$ and the Auger-like process ${ }^{21,22}$ have been analyzed.

More realistic, multicomponent, QD heterostructures have also been investigated. Several studies on the influence of the
QD environment on the electronic dynamics have been performed. The effects of optical and acoustical phonons, within the barrier and the matrix, on the QD electronic subsystem have also been demonstrated. ${ }^{8,9,23-25}$ Elastic Coulomb collisions of carriers in the wetting layer with those in the dots ${ }^{26}$ and charge fluctuations in the impurity state due to recharging through the free electron reservoir ${ }^{2}$ were also shown to affect the dynamics of QD optical transitions. Evidently, QD carriers will strongly interact not only with free charges in their environs but also with any nearby excitations which are accompanied by electric fields. It has been demonstrated ${ }^{27,28}$ on an InAs/GaAs QD heterostructure, that plasmons and plasmon phonons which reside in doped heterostructure components, are strongly coupled to the QD electronic subsystem. As a result, QD intraband carrier relaxation with a combination of emission from the substrate bulk and surface plasmon-LO-phonon modes will dominate relaxation processes at a distance of ca. $20 \mathrm{~nm}$ between the QD and the doped substrate.

To date, plasmon-induced relaxation processes in heterostructures based on ionic semiconductors have attracted most attention. However, covalent materials are of great interest for the fabrication of nanoelectronic devices. For example, $\mathrm{Ge} / \mathrm{Si} \mathrm{QD}$ heterostructures can easily be incorporated into existing $\mathrm{Si}$-based process technologies. Since the optical phonons of covalent semiconductor are not accompanied by electric fields they do not interact with plasmons. So, the only inherent source of the electric fields within such materials is from pure plasma oscillations. Therefore, an investigation of the relaxation processes induced by plasmons in doped covalent heterostructure layers is important with respect to both fundamental physics and applications.

In this paper we investigate the relaxation of hot carriers in QDs by scattering via interface plasmons (IPs) within the 


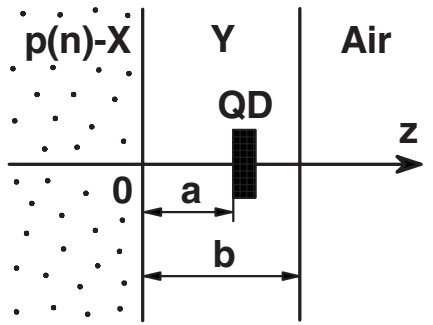

FIG. 1. Geometry of the double heterostructure. $a$ is the distance between the quantum dot and the doped covalent semiconductor $X$ $(X=\mathrm{Si}$ or $\mathrm{Ge}), b$ is the thickness of the undoped semiconductor layer $Y$ (not necessarily covalent, e.g., $Y=\mathrm{SiO}_{2}$ or $\mathrm{Si}_{x} \mathrm{Ge}_{1-x}$ ).

layered heterostructures. The paper is organized as follows. In Sec. II we develop a macroscopic model for IPs in double heterostructure. The Hamiltonian of the system in question, the dispersion law for IPs and the electric potential induced by the plasmons are derived. Section III is devoted to the calculation of the IP-assisted relaxation rate within a diskshaped QD. Our results show that the presence of scattering by interface plasmons contributes significantly to QD intraband relaxation and can, in some cases, determine the QD luminescence efficiency.

\section{THEORETICAL ANALYSIS OF THE INTERFACE PLASMON MODEL}

Without loss of generality, the double heterostructure which we will use as a model in order to investigate the coupling between a QD electronic subsystem and the IP modes is represented in Fig. 1. The heterostructure is grown along the $z$ axis. The interface is at $z=0$ and the surface is at $z=b$. The half-space $z<0$ (substrate) consists of a doped covalent semiconductor while the layer $0<z<b$ consists of undoped semiconductor or dielectric. The QD is located in the layer at a distance $a$ from the doped material. In this model, we assume the QD-IPs interaction does not appreciably perturb the energy spectra of the IPs or the QD. The QD will be considered only as a probe for the electric fields induced by the IPs. To calculate the IP eigenmodes and the corresponding electric potential at the dot location, we use an approach which has been used before for the description of the photon-plasmon interaction in a single heterostructure. ${ }^{29}$

Neglecting the retardation effect, let us start with the nonrelativistic Bloch equations describing the motion of free carriers and the electric field generated by their motion. These equations are ${ }^{30}$

$$
\begin{gathered}
\frac{\partial \mathbf{v}}{\partial t}=\nabla\left(\frac{e}{m} \phi-\frac{\mathbf{v}^{2}}{2}\right)-\frac{\nabla p}{m n}, \\
\frac{\partial n}{\partial t}=-\nabla(n \mathbf{v}), \\
\triangle \phi=\frac{4 \pi e}{\varepsilon}\left(n-n_{0}\right),
\end{gathered}
$$

where $\mathbf{v}(\mathbf{r}, t), n(\mathbf{r}, t)$, and $p(\mathbf{r}, t)$ are the hydrodynamic velocity, concentration, and pressure of the free carrier gas, $\phi(\mathbf{r}, t)$ is the self-consistent electric potential, $m$ is the effective mass of the carriers, $n_{0}$ is the space-independent dopant concentration and $\varepsilon$ is the dielectric constant. The system (1) is incomplete since it contains five equations and six unknown variables. Therefore, it must be supplemented by the state equation determining the correlation between pressure, concentration, and temperature of the carriers. In general, this correlation can be expressed only in an implicit form. ${ }^{31}$ If we restrict our description to only apply to a degenerate free carrier gas then the state equation reads ${ }^{29} p=\xi n^{5 / 3}$, where $\xi$ $=\left[\left(3 \pi^{2}\right)^{2 / 3} 5\right] \hbar^{2} / m$. Introducing the velocity potential $\psi(\mathbf{r}, t)$ according to the equation $\mathbf{v}=-\nabla \psi$ we can transform Eqs. (1),

$$
\begin{gathered}
\frac{\partial \psi}{\partial t}=\frac{1}{2}(\nabla \psi)^{2}-\frac{e}{m} \phi+\frac{5 \xi}{2 m} n^{2 / 3}, \\
\frac{\partial n}{\partial t}=\nabla(n \nabla \psi), \\
\triangle \phi=\frac{4 \pi e}{\varepsilon}\left(n-n_{0}\right) .
\end{gathered}
$$

System (2) can be linearized in the usual way by substitution of the following series expansion: $n(\mathbf{r}, t)=n_{0}+n_{1}(\mathbf{r}, t)$ $+n_{2}(\mathbf{r}, t)+\cdots, \quad \psi(\mathbf{r}, t)=\psi_{1}(\mathbf{r}, t)+\psi_{2}(\mathbf{r}, t)+\cdots$, and $\phi(\mathbf{r}, t)$ $=\phi_{0}+\phi_{1}(\mathbf{r}, t)+\phi_{2}(\mathbf{r}, t)+\cdots$. We suppose that the free carrier gas has a uniform concentration $n_{0}$ in the nonperturbed state. To a first approximation, we obtain the linear system of coupled equations,

$$
\begin{gathered}
\frac{\partial \psi_{1}}{\partial t}=-\frac{e}{m} \phi_{1}+\frac{\beta^{2}}{n_{0}} n_{1}, \\
\frac{\partial n_{1}}{\partial t}=n_{0} \triangle \psi_{1}, \\
\triangle \phi_{1}=\frac{4 \pi e}{\varepsilon} n_{1} .
\end{gathered}
$$

Here $\beta=\left(5 \xi n_{0}^{2 / 3} / 3 m\right)^{1 / 2}$ is the speed of propagation of hydrodynamic disturbance in the carrier gas. The system (3) is a set of the Euler equations for the Lagrangian

$$
\begin{aligned}
L \equiv \int d^{3} r \mathcal{L} \equiv & \int d^{3} r\left(\frac{\varepsilon}{8 \pi}\left(\nabla \phi_{1}\right)^{2}+m n_{1} \frac{\partial \psi_{1}}{\partial t}-\frac{1}{2} m n_{0}\left(\nabla \psi_{1}\right)^{2}\right. \\
& \left.+e \phi_{1} n_{1}-\frac{m \beta^{2}}{2 n_{0}} n_{1}^{2}\right),
\end{aligned}
$$

and the Hamiltonian corresponding to Eqs. (3) is given by

$$
\begin{aligned}
H & =\int d^{3} r\left(\frac{\partial \psi_{1}}{\partial t} \frac{\partial \mathcal{L}}{\partial\left(\partial \psi_{1} / \partial t\right)}-\mathcal{L}\right) \\
& =\int d^{3} r\left(-\frac{\varepsilon}{8 \pi}\left(\nabla \phi_{1}\right)^{2}+\frac{1}{2} m n_{0}\left(\nabla \psi_{1}\right)^{2}-e \phi_{1} n_{1}+\frac{m \beta^{2}}{2 n_{0}} n_{1}^{2}\right) .
\end{aligned}
$$

From now on, the subscript 1 will be omitted for clarity. 
In order to find the IP eigenmodes, their dispersion, and the self-consistent electrostatic potential induced by the plasmons, one needs to solve the system (3) with appropriate boundary conditions. In order to do this, we applied Eqs. (3) to every region within the heterostructure, solving the equations for doped $(d)$ and undoped $(u)$ materials, as well as for air $(a)$. The boundary conditions used have a clear physical meaning. First of all, we require the functions $\psi(\mathbf{r}, t), \phi(\mathbf{r}, t)$, and $n(\mathbf{r}, t)$ to be bounded across the whole space. The standard boundary conditions of classical electrodynamics should be also fulfilled. In particular, the electrostatic potential and the normal component of electric displacement must be continuous at the interface, $z=0$, and the surface, $z=b$, of the heterostructure: $\left.\phi_{d}\right|_{z=0}=\left.\phi_{u}\right|_{z=0},\left.\quad \phi_{u}\right|_{z=b}=\left.\phi_{a}\right|_{z=b}$, $\varepsilon_{d} d \phi_{d} /\left.d z\right|_{z=0}=\varepsilon_{u} d \phi_{u} /\left.d z\right|_{z=0}, \quad \varepsilon_{u} d \phi_{u} /\left.d z\right|_{z=b}=d \phi_{a} /\left.d z\right|_{z=b}$. Finally, the interface is impermeable for carriers. So, at the plane $z=0$, the normal component of the hydrodynamic velocity is zero $\left(d \psi_{d} /\left.d z\right|_{z=0}=0\right)$.

According to the symmetry of the system, we suppose that all the dynamic variables $a(\mathbf{r}, t)=\{\psi(\mathbf{r}, t), n(\mathbf{r}, t)$, $\phi(\mathbf{r}, t)\}$ have the form $a(\mathbf{r}, t)=a(z) \exp (i \mathbf{q} \mathbf{x}-i \omega t)$, where $\mathbf{q}$ is the two-dimensional wave vector, $\mathbf{x}$ is the radius vector in the interface plain, and $\omega$ is the frequency of the IPs. Some algebraic manipulation leads to the following result:

$$
\begin{gathered}
\phi(z)=i \frac{m \omega}{e} \chi(z)+\frac{4 \pi e}{\varepsilon_{d}} \frac{n(z)}{\Gamma_{q}^{2}-q^{2}}, \\
\psi(z)=\chi(z)-i \frac{\omega}{n_{0}} \frac{n(z)}{\Gamma_{q}^{2}-q^{2}},
\end{gathered}
$$

where $\chi(z)$ and $n(z)$ are solutions of the equations

$$
\begin{aligned}
& \frac{\partial n(z)}{\partial z}-\Gamma_{q}^{2} n(z)=0, \\
& \frac{\partial \chi(z)}{\partial z}-q^{2} \chi(z)=0,
\end{aligned}
$$

$\Gamma_{q}^{2}=\left(\omega_{p}^{2}+\beta^{2} q^{2}-\omega^{2}\right) / \beta^{2}, \omega_{p}=\left(4 \pi n_{0} e^{2} / \varepsilon_{d} m\right)^{1 / 2}$ is the plasma frequency. Evidently, the IP modes are near the interface between the doped and undoped materials if $\Gamma_{q}^{2}>0$. Using the above-listed boundary conditions, it is easy to obtain the dispersion relation for the IPs,

$$
\omega_{s}(q)=\left[\omega_{p}^{2}\left(1-\nu_{q}\right)+\frac{\beta^{2} q^{2}}{2}+\beta q\left(\frac{\beta^{2} q^{2}}{4}+\omega_{p}^{2} \nu_{q}\right)^{1 / 2}\right]^{1 / 2},
$$

where

$$
\begin{gathered}
\nu_{q}=\frac{\varepsilon_{u} \eta_{-}}{\varepsilon_{u} \eta_{-}+\varepsilon_{d} \eta_{+}}, \\
\eta_{ \pm}=1 \pm \frac{\varepsilon_{u}-1}{\varepsilon_{u}+1} e^{-2 q b}
\end{gathered}
$$

Notice that Eq. (5) for $b \rightarrow \infty$ and $\varepsilon_{d}=\varepsilon_{u}=1$ transforms to Eq. (41) of Ref. 29.

Figure 2 (left-hand panel) illustrates the dispersion rela-
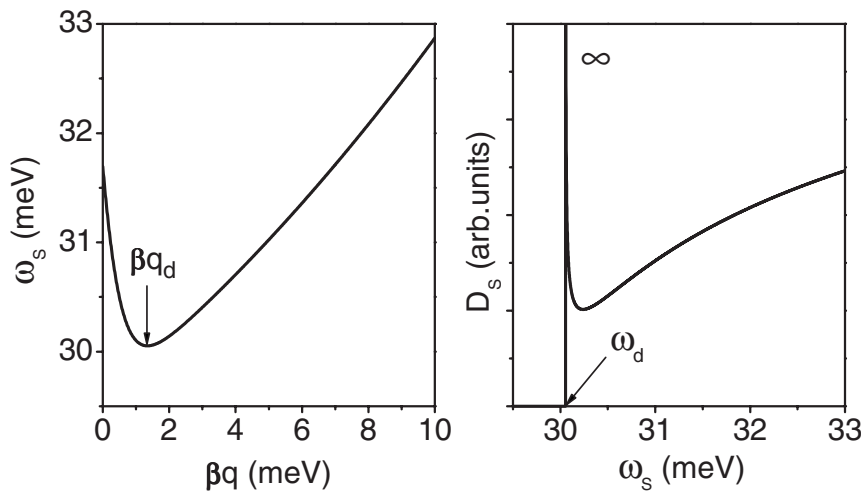

FIG. 2. Left-hand panel: The dispersion relation $\omega_{s}(q)$ for the IP modes in the double $p-\mathrm{Si} / \mathrm{SiO}_{2} /$ air heterostructure (Fig. 1). The concentration of free holes $n_{0}=5 \times 10^{18} \mathrm{~cm}^{-3}$, the thickness of the $\mathrm{SiO}_{2}$ layer $b=50 \mathrm{~nm}$. Right-hand panel: The density of states corresponding to $\omega_{s}(q)$. The symbol $\infty$ marks the critical point at which the DOS diverges.

tion for the $p-\mathrm{Si} / \mathrm{SiO}_{2} /$ air heterostructure. In the calculations below, we consider a $p$-doped substrate $(p-\mathrm{Si})$ with a free hole concentration $n_{0}$. It is apparent that $\omega_{s}(q)$ has a minimum at a nonzero value of the IP wave vector $q_{d}$. This results from the existence of the second interface $\left(\mathrm{SiO}_{2} /\right.$ air $)$ between the media with different dielectric constants. The physical origin of the local minimum is the interference between the electric field penetrating through the doped semiconductor/dielectric interface and the electric field reflected from the air/dielectric boundary. The depth, $\Delta$ $=\omega_{s}(0)-\omega_{s}\left(q_{d}\right)$ and position, $q_{d}$ of the minimum depends on the undoped layer thickness, $b$ (Fig. 3). The critical points of the quasiparticle energy spectra occur where $\nabla_{q} \omega(q)=0$. In this case the critical points form circles with radii $q_{d}$, since the function $\omega_{s}(q)$ is isotropic in $q$ space. These critical points $\mathbf{q}_{d}$ of the energy spectra result in the appearance of critical points $\omega_{d}=\omega_{s}\left(\mathbf{q}_{d}\right)$ in the IP density of states (DOS),

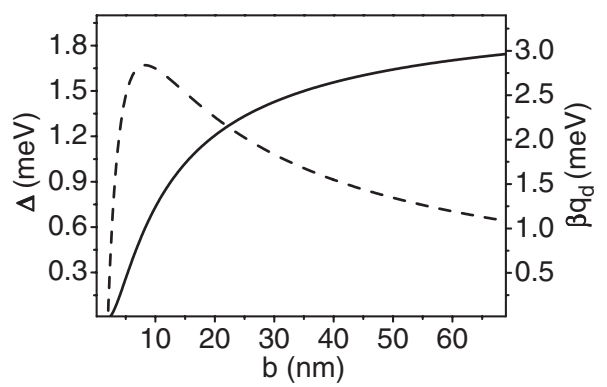

FIG. 3. Solid line (left-hand scale): Depth of the energy dispersion $\omega_{s}(q)$ minimum in the double $p-\mathrm{Si} / \mathrm{SiO}_{2} /$ air heterostructure (Fig. 1) as a function of the thickness $b$ of the $\mathrm{SiO}_{2}$ layer. Dashed line (right-hand scale): Position of the minimum as a function of the thickness $b$. In both cases the density of free holes is $5 \times 10^{18} \mathrm{~cm}^{-3}$. 


$$
\begin{aligned}
D_{s}(\omega) & =\frac{1}{(2 \pi \hbar)^{2}} \int_{0}^{\infty} \delta\left[\omega-\omega_{s}(q)\right] q^{2} d q \\
& =\sum_{j}\left(\frac{q(\omega)}{2 \pi \hbar}\right)^{2}\left|\frac{\partial q(\omega)}{\partial \omega}\right|
\end{aligned}
$$

where the summation is extended over all $j$ branches of the function $q(\omega)$. It is significant that in the case of a single heterostructure there are no critical points of this type in the IP DOS. As one can readily see from Eq. (5), in the limiting case as $b \rightarrow \infty$, the dispersion of IP modes is a steadily increasing function. The IP DOS corresponding to the dispersion relation $\omega_{s}(q)$ plotted in Fig. 2 (left-hand panel) is shown on the right-hand panel of Fig. 2. A simple analysis of Eq. (6) shows that the DOS diverges as $\left(\omega-\omega_{d}\right)^{-1 / 2}$ at the critical point $\omega_{d}$. This behavior of the DOS is typical for one-dimensional systems. ${ }^{32}$ Obtaining the same result for the two-dimensional problem is not surprising because the isotropy of the IP dispersion reduces the dimension of the system to unity. Obviously, the divergence of the DOS in some way should be attributed to IP-assisted processes.

Let us use the solution of the system (3) for the secondary quantization of the fields involved in the Hamiltonian (4) and introduce the IP excitations with the creation (annihilation) operator $\hat{b}_{q}^{+}\left(\hat{b}_{q}\right)$. The Hamiltonian (4) takes the form

$$
\hat{H}=\sum_{\mathbf{q}} \hbar \omega_{s}(q)\left(\hat{b}_{q}^{+} \hat{b}_{q}+1 / 2\right),
$$

and the self-consistent electric potential of the IP modes can be represented as

$$
\hat{\phi}_{s}(\mathbf{r})=\sum_{\mathbf{q}}\left[\phi_{q}(z) e^{i \mathbf{q} \mathbf{x}} \hat{b}_{q}+\phi_{q}^{*}(z) e^{-i \mathbf{q} \mathbf{x}} \hat{b}_{q}^{+}\right]
$$

where

$$
\phi_{q}(z)=\frac{4 \pi e \nu_{q}}{\varepsilon_{u} \eta_{-}}\left(\frac{\hbar n_{0}}{m \omega \sigma q} \frac{\Gamma_{q}}{2 \Gamma_{q}+q}\right)^{1 / 2} \begin{cases}{\left[\left(q \varepsilon_{u} \eta_{-}+\Gamma_{q} \varepsilon_{d} \eta_{+}\right) e^{q z}-q\left(\varepsilon_{u} \eta_{-}+\varepsilon_{d} \eta_{+}\right) e^{\Gamma_{q}}\right] /\left[\varepsilon_{d}\left(\Gamma_{q}-q\right)\right],} & z \leq 0, \\ e^{-q z}+\left(\eta_{+}-1\right) e^{q z}, & 0<z \leq b \\ 2 \varepsilon_{u} /\left(\varepsilon_{u}+1\right) e^{-q z} & z>b,\end{cases}
$$

$\omega \equiv \omega_{s}(q), \sigma$ is the normalization area.

The coupling between the IP modes and any charged excitations (e.g., electrons or holes) can be expressed by the operator $e \hat{\phi}_{s}(\mathbf{r})$. This interaction gives rise to the IP-assisted light absorption, luminescence, and Raman scattering, as well as the intraband carrier relaxation. As an example, the relaxation process developing in the QD electronic subsystem will be considered in the next section.

\section{QUANTUM DOT INTRABAND CARRIER RELAXATION}

The results obtained in the preceding section allow us to estimate the QD intraband relaxation rate due to interaction with the doped substrate of the double heterostructure (Fig. 1) via the electric potential (7) induced by the IP modes. This coupling results in transitions between the initial $|i\rangle$ and final $|f\rangle$ states of the electron (hole) upon emission or absorption of the IP. Assuming that temperatures are sufficiently small, $\omega_{s}(q)>>k_{B} T$, we can restrict our analysis to relaxation processes with emission of IP quanta. Then, to a first approximation, the rates of the intraband transitions as a function of the intraband QD level spacing $\Omega=\left(E_{i}-E_{f}\right) / \hbar$ are given by

$$
W_{s}(\Omega)=\frac{2 \pi}{\hbar^{2}} \sum_{\mathbf{q}}\left|\left\langle i\left|e \phi_{q}(z) e^{i \mathbf{q} \mathbf{x}}\right| f\right\rangle\right|^{2} e^{-2 q a} \delta\left[\Omega-\omega_{s}(q)\right],
$$

where the origin is chosen at the QD position. Generally, the initial, final or both QD states are degenerate in some quantum numbers which will be marked below by a prime. To take into account the degeneration in Eq. (8), one should average the right-hand side of this expression over the degenerate initial states $\left|i i^{\prime}\right\rangle$ and sum over degenerate final states $\left|f f^{\prime}\right\rangle$. As a result, the following expression for the intraband relaxation rate will be obtained:

$$
W_{s}(\Omega)=\frac{2 \pi}{\hbar^{2}} \sum_{\mathbf{q}} \Phi_{\mathbf{q}}(\Omega) e^{-2 q a} \delta\left[\Omega-\omega_{s}(q)\right],
$$

where the function

$$
\Phi_{\mathbf{q}}(\Omega)=\sum_{f}\left\langle\left|\left\langle i i^{\prime}\left|e \phi_{q}(z) e^{i \mathbf{q} \mathbf{x}}\right| f f^{\prime}\right\rangle\right|^{2}\right\rangle_{i}
$$

contains all the information on the QD parameters. Since Eq. (9) is similar in its mathematical structure to the IP DOS [Eq. (6)], the relaxation rate $W_{s}(\Omega)$ will diverge at the DOS critical points. The simplest way to avoid this problem is to replace the $\delta$ function in Eq. (9) by the phenomenological Lorenzian

$$
\delta\left[\Omega-\omega_{s}(q)\right]-\rightarrow \frac{1}{\pi} \frac{\gamma}{\left[\Omega-\omega_{s}(q)\right]^{2}+\gamma^{2}},
$$

where $\gamma=1 / 2\left(\gamma_{i}+\gamma_{f}+\gamma_{i p}\right)+\gamma_{p d}$ is the total dephasing rate of the transition between the initial and final QD states, $\gamma_{i}, \gamma_{f}$, and $\gamma_{i p}$ are the inverse lifetimes of electron (hole) states $(i$ and $f$ ) and IP excitations (ip), $\gamma_{p d}$ is the pure dephasing rate, $\gamma$ is the phenomenological parameter which is assumed to be determined by relaxation processes occurring in addition to the IP-assisted relaxation. In order to further analyze the intraband carrier relaxation, let us consider a cylindrical quan- 

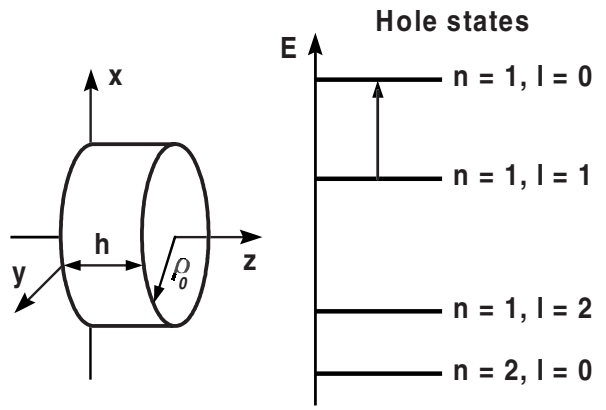

FIG. 4. Cylindrically symmetric model quantum dot. Level $|2\rangle$ decays to the ground state $|1\rangle$ by spontaneous emission of IP.

tum dot model (Fig. 4), a model which is frequently used for real QD systems. ${ }^{33-35}$ We will examine a strong confinement regime when the height $(h)$ and radius $\left(\rho_{0}\right)$ of the QD are smaller than the exciton Bohr radius of the QD bulk material. In order to be definitive, we will consider the intraband relaxation of holes although similar results can be obtained for the electronic relaxation as well. The energy spectrum and wave functions of holes in a cylindrically symmetric QD providing an infinite potential barrier have the form

$$
\begin{gathered}
|k n l\rangle=\left(\frac{2}{\pi h \rho_{0}^{2}}\right)^{1 / 2} \frac{J_{l}\left(\zeta_{l n} \rho / \rho_{0}\right)}{J_{l+1}\left(\zeta_{l n}\right)} \sin \left(\frac{\pi k}{h} z\right) e^{i l \varphi}, \\
E_{k n l}=\frac{\hbar^{2}}{2 m^{*}}\left(\frac{\pi^{2} k^{2}}{h^{2}}+\frac{\zeta_{l n}^{2}}{\rho_{0}^{2}}\right),
\end{gathered}
$$

where $k \in N, \zeta_{l m}$ is the $n$th zero of the Bessel function of the $l$ th order $\left[J_{l}\left(\zeta_{l n}\right)=0\right], m^{*}$ is the hole effective mass. Utilizing Eq. (10) for calculating the function $\Phi_{\mathbf{q}}$ one may obtain

$$
\Phi_{\mathbf{q}}(\Omega)=2 B\left(l_{f}\right)\left(\frac{e \phi_{q}}{\eta_{+}} T_{k_{f}}^{k_{i}}(q) I_{k_{f}}^{k_{i}}(q) J_{l_{f_{f}} n_{f} n_{i}}^{n_{n}}(q)\right)^{2},
$$

where $B\left(l_{f}\right)=1$ for $l_{f}=0$ and $B\left(l_{f}\right)=2$ for other cases,

$$
\begin{gathered}
T_{k_{f}}^{k_{i}}(q)=\left[1+(-1)^{k_{f}+k_{i}}\left(\eta_{+}-1\right) e^{q(h+2 a)}\right] \\
I_{k_{f}}^{k_{i}}(q)=\frac{4 \pi^{2} q h\left[1-(-1)^{k_{f}+k_{i}} e^{-q h}\right] k_{f} k_{i}}{\left[q^{2} h^{2}+\pi^{2}\left(k_{f}-k_{i}\right)^{2}\right]\left[q^{2} h^{2}+\pi^{2}\left(k_{f}+k_{i}\right)^{2}\right]}, \\
J_{l_{f} n_{f} n_{i}}(q)=2 \int_{0}^{1} d x x \frac{J_{l_{f}}\left(\zeta_{l_{f} n_{f}} x\right) J_{l_{i}}\left(\zeta_{l_{i} n_{i}} x\right) J_{l_{i}-l_{f}}\left(q \rho_{0} x\right)}{J_{l_{f}+1}\left(\zeta_{l_{f} n_{f}}\right) J_{l_{i}+1}\left(\zeta_{l_{i} n_{i}}\right)} .
\end{gathered}
$$

The hole intraband relaxation rate is given by

$$
\begin{aligned}
W_{s}(\Omega)= & B\left(l_{f}\right) \frac{m \omega_{p}^{4}}{\pi \hbar n_{0}}\left(\frac{\varepsilon_{d}}{\varepsilon_{u}}\right)^{2} \int_{0}^{\infty} \frac{d q}{\omega} \frac{\gamma}{(\Omega-\omega)^{2}+\gamma^{2}} \\
& \times \frac{\Gamma_{q}}{2 \Gamma_{q}+q}\left(\frac{\nu_{q}}{\eta_{-}} T_{k_{f}}^{k_{i}}(q) I_{k_{f}}^{k_{i}}(q) J_{l_{f} n_{f}}^{l_{i} n_{i}}(q) e^{-q a}\right)^{2},
\end{aligned}
$$

where $\omega \equiv \omega_{s}(q)$.

In order to illustrate the mechanism of intraband hole relaxation in a double heterostructure (Fig. 1), consider a Ge QD with a fixed height, $h=5 \mathrm{~nm}$. We only consider the two lowest energy hole states $|1\rangle=|110\rangle$ and $|2\rangle=|111\rangle$ (see Fig.

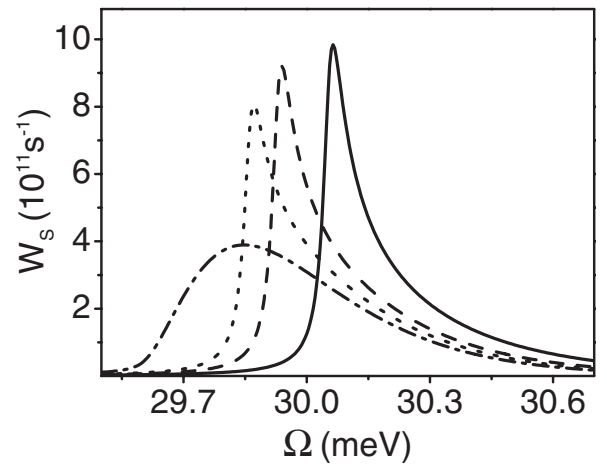

FIG. 5. The hole relaxation rates as a function of the energy level spacing $\Omega$ of the intraband transition $|2\rangle \rightarrow|1\rangle$ for various undoped layer thicknesses $b$ : the solid line, $b=50 \mathrm{~nm}$; the dashed line, $b=75 \mathrm{~nm}$; and the dotted line, $b=100 \mathrm{~nm}$. The dashed-dotted line shows the corresponding relaxation rates for a single heterostructure. In the calculations the following parameters were used: $a=40 \mathrm{~nm}, n_{0}=5 \times 10^{18} \mathrm{~cm}^{-3}$, and $\gamma=0.02 \mathrm{meV}$.

4). In all our calculations, the effective mass of heavy holes for Ge QDs has been used $\left(m^{*}=0.352 m_{e}\right)$. The relationship $\rho_{0}=\left[\hbar\left(\zeta_{2}^{2}-\zeta_{1}^{2}\right) /\left(2 m^{* 2} \Omega\right)\right]^{1 / 2}$ between the QD radius and $\Omega$ was employed to take account of variations in the energy level spacing with QD size. If the QD size is fixed and the energy level spacing is varied by other means, e.g., by arbitrarily changing $\Omega$ in the equations, qualitatively similar spectra for the relaxation rates have been obtained. It should be noted that the model of a QD with infinite potential barriers is reasonable for $\mathrm{Ge}$ dots embedded in an $\mathrm{SiO} 2$ matrix, a system with large band offsets.

Figure 5 shows the relaxation rate spectra for different $b$ for the $|2\rangle \rightarrow|1\rangle$ transition and the same spectrum for a single heterostructure. One can see that the relaxation window corresponding to the energy dispersion relation $\omega_{s}(q)$ of the IP modes is opened. Relaxation within the window is rapid: the relaxation rates exceed $10^{10} \mathrm{~s}^{-1}$ for energies of about $1 \mathrm{meV}$. We assumed in calculations that the transition dephasing rate $\gamma$ was equal to $0.02 \mathrm{meV}$.

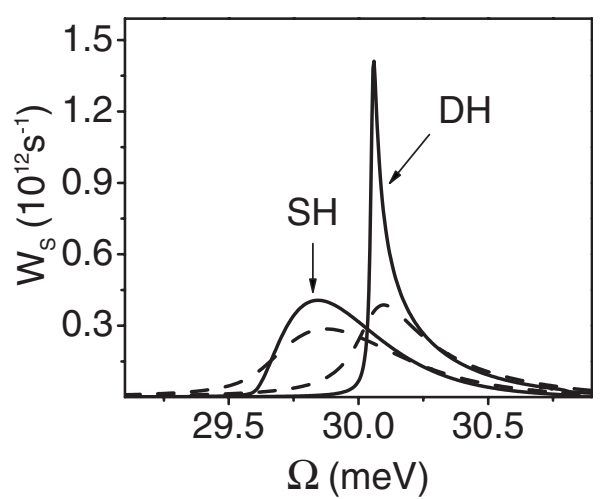

FIG. 6. Hole relaxation rates as a function of the energy level spacing $\Omega$ of the intraband transition $|2\rangle \rightarrow|1\rangle$ for different dephasing rates $\gamma$ : for the solid lines, $\gamma=0.01 \mathrm{meV}$; the dashed lines, $\gamma$ $=0.1 \mathrm{meV}$. The symbols $\mathrm{SH}$ and $\mathrm{DH}$ indicate single and double heterostructures, respectively. In calculations the following parameters were used: $a=40 \mathrm{~nm}, b=50 \mathrm{~nm}$, and $n_{0}=5 \times 10^{18} \mathrm{~cm}^{-3}$. 


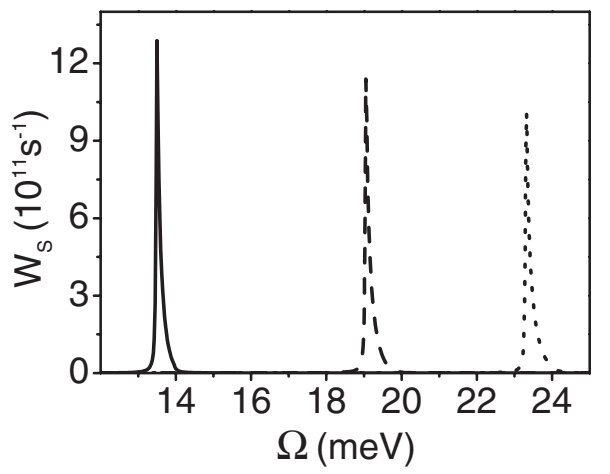

FIG. 7. Hole relaxation rates as a function of level spacing $\Omega$ of the intraband transition $|2\rangle \rightarrow|1\rangle$ for different free carrier concentrations $n_{0}$ : for the solid line, $n_{0}=10^{18} \mathrm{~cm}^{-3}$; the dashed line, $n_{0}=2$ $\times 10^{18} \mathrm{~cm}^{-3}$; and the dotted line, $n_{0}=3 \times 10^{18} \mathrm{~cm}^{-3}$. In these calculations the following parameters were used: $a=40 \mathrm{~nm}, b=50 \mathrm{~nm}$, and $\gamma=0.02 \mathrm{meV}$.

It is important to note the following three differences between double and single heterostructures. First, the existence of the critical points in the IP DOS for the double heterostructure results in a strong enhancement of the intraband relaxation rates as compared to those in the single heterostructure. Second, narrowing of the spectral width of the relaxation window occurs. Finally, the spectral position of the relaxation window is shifted towards higher energies with decreasing undoped layer thickness. Undoubtedly, the enhancement value depends on the transition dephasing rate $\gamma$. Although reliable data on $\gamma$ are lacking, we can estimate its lower limit. In accordance with the results obtained by several research groups, ${ }^{36-40}$ the electron (hole) dephasing rates in QDs at low temperatures vary from several $\mu \mathrm{eV}$ to several tens of $\mu \mathrm{eV}$. On the other hand, the inverse lifetime $\left(\gamma_{i p}\right)$ of the IP modes contribute to $\gamma$ additively. Its value is unknown a priori. In order to clarify this problem, we calculated the hole intraband relaxation rates with a $\gamma$ value of $100 \mu \mathrm{eV}$, assuming that $\gamma$ was determined by $\gamma_{i p}$ (see Fig. 6). One can see that, even for this case, distinct enhancement of the intraband relaxation rates in the double heterostructure takes place. Notwithstanding these significant differences, the intraband relaxation rates in double heterostructures exhibit $n_{0}$ and $a$ dependence similar to those for a single heterostructure. Figures 7 and 8 summarize how the spectral position of the relaxation window changes with $n_{0}$ and the relaxation rates increase with decreasing $a$.

\section{CONCLUSION}

We have examined the scattering of IPs at the interface of doped covalent semiconductor substrates in a double heterostructure and the associated relaxation processes in the QD embedded in an adjacent undoped layer within the heterostructure. Using a hydrodynamic approach, a simple estima-

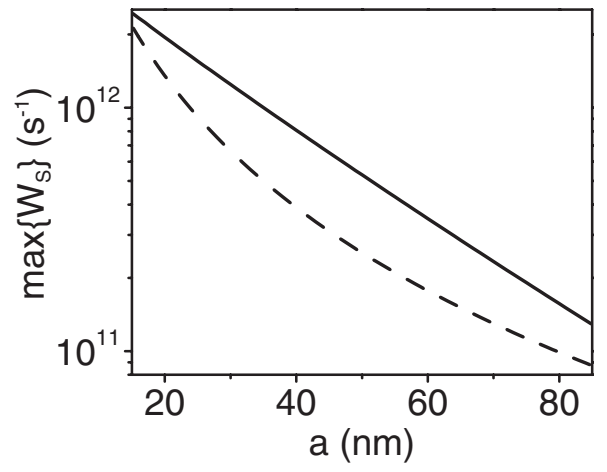

FIG. 8. Maximum hole relaxation rates for the transition $|2\rangle$ $\rightarrow|1\rangle$ as a function of the distance $a$ between the QD and the doped substrate. Solid line - the double heterostructure with $b=100 \mathrm{~nm}$; dashed line - the single heterostructure. $n_{0}=5 \times 10^{18} \mathrm{~cm}^{-3}, \gamma$ $=0.02 \mathrm{meV}$.

tion of the IP-induced relaxation of a QD electronic subsystem, including the effect of the finite lifetime of the IP modes, has been presented. It has been shown that the IP DOS has critical points arising from the presence of the undoped layer. The positions of these critical points depend on the layer thickness. Their presence results in enhancement of IP-assisted relaxation processes in QDs. We have demonstrated that QD intraband carrier relaxation is enhanced for layer thicknesses of the order of several tens of nanometers. This relaxation mechanism, inherent to doped covalent semiconductors, is technologically important for a variety of nanostructure-based devices, where the QD layer is located in the immediate vicinity of the $p(n)$-doped elements within the heterostructure and covered with a thin cap layer. The concentration, distance, and layer thickness dependencies of the relaxation rates offer the opportunity to engineer QD electronic dynamics in doped Si-based heterostructures. An experimental verification of the relaxation mechanism discussed in covalent QD heterostructures is required. In directgap InAs/GaAs QD heterostructures, an analysis of the resonant photoluminescence spectra of QDs can be used to demonstrate that plasmons and plasmon phonons, resident in the doped heterostructure components, can dominate the relaxation processes. ${ }^{27}$ The concentration, distance, and layer thickness dependencies of the relaxation rates for the nondirect gap QD heterostructures considered in our study can be determined from an analysis of the risetime of the phononassisted resonant photoluminescence. Experiments to explore this are presently underway.

\section{ACKNOWLEDGMENTS}

Three of the authors (A.V.F., A.V.B., I.D.R.) are grateful to the RFBR (Grant Nos. 06-02-17036a and 05-02-16212a) for partial financial support of this work. One of the authors (I.D.R.) is also thankful to the Dynasty Foundation for a research grant. 
*a_v_fedorov@inbox.ru

${ }^{1}$ L. Guo, E. Leobandung, and S. Chou, Science 275, 649 (1997).

${ }^{2}$ T. Itakura and Y. Tokura, Phys. Rev. B 67, 195320 (2003).

${ }^{3}$ K. Yano, T. Ishii, T. Sano, T. Mine, F. Murai, T. Hashimoto, T. Koboyashi, T. Kure, and K. Seki, Proc. IEEE 87, 633 (1999).

${ }^{4}$ M. Dutta and M. A. Stroscio, Advances in Semiconductor Lasers and Applications to Optoelectronics (World Scientific, Singapore, 2000).

${ }^{5}$ I. V. Ignatiev, I. E. Kozin, V. G. Davydov, S. V. Nair, J.-S. Lee, H.-W. Ren, S. Sugou, and Y. Masumoto, Phys. Rev. B 63, 075316 (2001).

${ }^{6}$ X.-Q. Li and Y. Arakawa, Phys. Rev. B 57, 12285 (1998).

${ }^{7}$ X.-Q. Li, H. Nakayama, and Y. Arakawa, Phys. Rev. B 59, 5069 (1999).

${ }^{8}$ F. Gindele, K. Hild, W. Langbein, and U. Woggon, Phys. Rev. B 60, R2157 (1999).

${ }^{9}$ A. V. Baranov, V. Davydov, H.-W. Ren, S. Sugou, and Y. Masumoto, J. Lumin. 87-89, 503 (2000).

${ }^{10}$ I. V. Ignatiev, I. E. Kozin, S. V. Nair, H.-W. Ren, S. Sugou, and Y. Masumoto, Phys. Rev. B 61, 15633 (2000).

${ }^{11}$ T. Inoshita and H. Sakaki, Phys. Rev. B 46, 7260 (1992).

${ }^{12}$ R. Heitz, M. Veit, N. N. Ledentsov, A. Hoffmann, D. Bimberg, V. M. Ustinov, P. S. Kop'ev, and Z. I. Alferov, Phys. Rev. B 56, 10435 (1997).

${ }^{13}$ P. C. Sercel, Phys. Rev. B 51, 14532 (1995).

${ }^{14}$ D. F. Schroeter, D. J. Griffiths, and P. C. Sercel, Phys. Rev. B 54, 1486 (1996).

${ }^{15}$ X.-Q. Li and Y. Arakawa, Phys. Rev. B 56, 10423 (1997).

${ }^{16}$ P. A. Knipp and T. L. Reinecke, Phys. Rev. B 46, 10310 (1992).

${ }^{17}$ G. Biese, C. Schüller, K. Keller, C. Steinebach, D. Heitmann, P. Grambow, and K. Eberl, Phys. Rev. B 53, 9565 (1996).

${ }^{18}$ S. Zanier, Y. Guldner, J. P. Vieren, G. Faini, E. Cambril, and Y. Campidelli, Phys. Rev. B 57, 1664 (1998).

${ }^{19}$ O. Verzelen, R. Ferreira, and G. Bastard, Phys. Rev. B 62, R4809 (2000).
${ }^{20}$ S. Sauvage, P. Boucaud, R. P. S. M. Lobo, F. Bras, G. Fishman, R. Prazeres, F. Glotin, J. M. Ortega, and J.-M. Gerard, Phys. Rev. Lett. 88, 177402 (2002).

${ }^{21}$ U. Bockelmann and T. Egeler, Phys. Rev. B 46, 15574 (1992).

${ }^{22}$ A. L. Efros, V. A. Kharchenko, and M. Rosen, Solid State Commun. 93, 281 (1995).

${ }^{23}$ U. Bockelmann and G. Bastard, Phys. Rev. B 42, 8947 (1990).

${ }^{24}$ H. Benisty, Phys. Rev. B 51, 13281 (1995).

${ }^{25}$ A. V. Fedorov, A. V. Baranov, and Y. Masumoto, Solid State Commun. 122, 139 (2002).

${ }^{26}$ A. V. Uskov, K. Nishi, and R. Lang, Appl. Phys. Lett. 74, 3081 (1999).

${ }^{27}$ A. V. Baranov, A. V. Fedorov, I. D. Rukhlenko, and Y. Masumoto, Phys. Rev. B 68, 205318 (2003).

${ }^{28}$ A. V. Fedorov, A. V. Baranov, I. D. Rukhlenko, and Y. Masumoto, Solid State Commun. 128, 219 (2003).

${ }^{29}$ R. H. Ritchie and R. Wilems, Phys. Rev. 178, 372 (1969).

${ }^{30}$ I. V. Tokatly and O. Pankratov, Phys. Rev. B 62, 2759 (2000).

${ }^{31}$ A. V. Fedorov, A. V. Baranov, I. D. Rukhlenko, and S. V. Gaponenko, Phys. Rev. B 71, 195310 (2005).

${ }^{32}$ F. Bassani and G. P. Parravicini, Electronic States and Optical Transitions in Solids (Pergamon, London, 1975).

${ }^{33}$ S. Le Goff and B. Stébé, Phys. Rev. B 47, 1383 (1993).

${ }^{34}$ J. Song and S. E. Ulloa, Phys. Rev. B 52, 9015 (1995).

${ }^{35}$ P. Matagne and J.-P. Leburton, Phys. Rev. B 65, 235323 (2003).

${ }^{36}$ D. Gammon, E. Snow, E. Shanabrook, D. Katzer, and D. Park, Science 273, 87 (1996).

${ }^{37}$ L. Besombes, K. Kheng, L. Marsal, and H. Mariette, Phys. Rev. B 63, 155307 (2001).

${ }^{38}$ P. Borri, W. Langbein, S. Schneider, U. Woggon, R. L. Sellin, D. Ouyang, and D. Bimberg, Phys. Rev. Lett. 87, 157401 (2001).

${ }^{39}$ M. Bayer and A. Forchel, Phys. Rev. B 65, 041308(R) (2002).

${ }^{40}$ A. V. Baranov, V. Davydov, A. V. Fedorov, M. Ikezawa, H.-W. Ren, S. Sugou, and Y. Masumoto, Phys. Rev. B 66, 075326 (2002). 